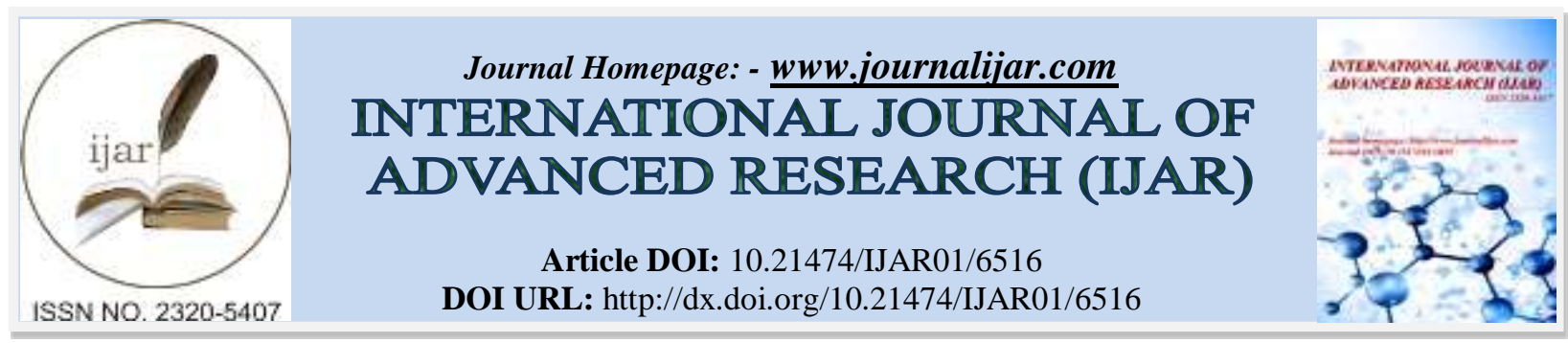

RESEARCH ARTICLE

\title{
PROGNOSTIC VALUE OF THE EXPRESSION OF ENDOGENOUS HYPOXIA ASSOCIATED PROTEINS HYPOXIA INDUCIBLE FACTOR-1 ALPHA (HIF-1A) AND CARBONIC ANHYDRASE ISOFORM 9 (CAIX) EXPRESSIONS IN BREAST CARCINOMA.
}

\author{
Ola A. Harb ${ }^{1}$, Ibtesam Elhasadi ${ }^{2}$, Amal M A Adim ${ }^{2}$, Warda M Said ${ }^{2}$, Sharifa A. M. El.Ammari ${ }^{2}$, Basant Sh El \\ Shafaay ${ }^{3}$ Fady M. Habib ${ }^{4}$ and Loay M. Gertallah ${ }^{4}$. \\ 1. Department of Pathology, Faculty of Medicine, Zagazig University, Zagazig 44519, Egypt. \\ 2. Department of Pathology, Faculty of Medicine, Benghazi University, Benghazi, Libya. \\ 3. Department of Clinical Oncology and nuclear medicine, Faculty of Medicine, Zagazig University, Zagazig \\ 44519, Egypt. \\ 4. Department of General surgery, Faculty of Medicine, Zagazig University, Zagazig 44519, Egypt.
}

\section{Manuscript Info}

Manuscript History

Received: 12 December 2017

Final Accepted: 14 January 2018

Published: February 2018

Key words:-

breast carcinoma, hypoxia; HIF-1 $\alpha$; CAIX; immunohistochemistry; prognosis.

\section{Abstract}

Background: hypoxia has been found to be related to malignant initiation, progression, increasing the occurrence of metastasis and therapy resistance in many cancer types, which made a real need for discovering drugs that could antagonize the bad effect of hypoxia in cancer, decide which patients will have benefit from such anti-hypoxia therapy then to monitor response to therapy, especially in breast carcinoma.

It is important to detect degree of hypoxia in each cancer that could be done by evaluation of the expression of hypoxia-associated proteins in cancer biopsies e.g. hypoxia inducible factor-1 alpha (HIF-1 $\alpha$ ) and carbonic anhydrase IX (CAIX) and their detailed role in breast cancer is still uncertain and gives conflicting results.

Aim of the work: was to evaluate HIF- $1 \alpha$ and CAIX expressions in breast carcinoma, correlating their expressions with each other, with presence of lymph node $\&$ distant metastases, with recurrence free and overall survival rates of female patients with breast cancer.

Methods: we evaluated HIF-1 $\alpha$ \& CAIX expressions in sections from 90 paraffin blocks of breast carcinoma using immunohistochemistry. We analyzed correlations between their levels of expressions, clinicpathological and prognostic parameters of our patients.

Results: HIF- $1 \alpha$ and CAIX positive expression in breast carcinoma was related to advanced stage, presence of lymph node metastases, HER2 amplified and triple negative molecular subtypes $(\mathrm{p}<0.001)$, higher tumor grade $(\mathrm{p}=0.001 \& 0.02$ respectively) and negative ER $(\mathrm{p}=$ $0.005 \& 0.008$ respectively) \& PR $(\mathrm{p}=0.009 \& 0.027$ respectively) hormonal receptors, The expression of both markers was significantly positively correlated with each other $(p<0.001)$. HIF- $1 \alpha$ and CAIX positive expression in breast carcinoma was associated with shortened recurrence free and overall survival rates $(\mathrm{p}<0.001)$.

Conclusion: HIF- $1 \alpha$ and CAIX are markers of poor prognosis of breast carcinoma patients. 


\section{Introduction:-}

Breast carcinoma is considered the commonest cancer type and the $2^{\text {nd }}$ leading cause of females' cancer related mortality [Lakhani et al., 2012]. Breast carcinoma lymph node and distant metastasis are the most important prognostic factor for patients [Sugie et al. 2013]. Because of perfusion deficits, solid tumors have heterogeneous regions of hypoxia (reduced pO2). Additionally, it has been reported that the altered tumor metabolism can also contribute to tumor hypoxia [Wojtkowiak et al. 2015]. Hypoxia has been found to be related to malignant initiation, progression, increasing the occurrence of metastasis and therapy resistance in many cancer types, also it has a prognostic marker of poor patients' survival rates [Wigerup et al., 2016]. The negative consequences of tumor hypoxia on cancer of various types, made a real need for discovering drugs that could antagonize the bad effect of hypoxia in cancer [Sun et al. 2012]. Also, hypoxia assessment in different cancer regions which can help to decide which patients will have benefit from such anti-hypoxia therapy then to monitor response to therapy, especially in breast carcinoma. An easy method of hypoxia detection could be done by assessment of endogenous hypoxiaassociated proteins expression in tumor biopsies using immunohistochemistry e.g. hypoxia inducible factor-1 alpha (HIF-1 $\alpha)$ and carbonic anhydrase IX (CAIX) [Bussink et al., 2003].

HIF-1 $\alpha$ protein is destroyed and removed within minutes in conditions of normal oxygen concentration, while it is stabilized and up regulated during hypoxia. When it is stabilized, it is translocated to the nucleus, activated and forming active transcription complex. After that it binds to hypoxia response element in promoters of different target genes that could allow increase in oxygen availability and/or increase metabolic adaptation to hypoxia [Semenza GL. 2010]. CAIX is a glycoprotein that is considered a major HIF-1 $\alpha$ downstream target; its expression has been related to prognosis in some types of cancer [Zatovicova et al. 2010]. So it is considered an attractive endogenous marker of detection of hypoxia and evaluating its role in cancer prognosis [Supuran, 2008]. Relation between HIF$1 \alpha$ and CAIX expression in cancer cells, the underlying mechanism of actions of both markers and their roles in induction by hypoxia remain unclear. Although several studies have evaluated expression of both markers in many cancers including breast carcinoma but up till now no accurate role has been detected regarding their clinicopathological and prognostic role in breast carcinoma patients [Wigerup et al., 2016] and also, there is no previous studies that have studied them both in a large number of Egyptian females.

Aim of the work; was to evaluate HIF- $1 \alpha$ and CAIX expressions in breast carcinoma, correlating their expressions with each other, with presence of lymph node \& distant metastases, with recurrence free and overall survival rates of female patients with breast cancer.

\section{Patients and methods:-}

We started our prospective cohort study in July 2014 finished it in July 2017, where we included ninety female patients that are having breast carcinoma that were admitted to general surgery department oncology unit, faculty of medicine Zagazig university, Zagazig Egypt, where mastectomy were surgeons that are sharing in the study performed modified radical mastectomy with axillary clearance for all cases, then sent the biopsies to Pathology Department, Faculty of Medicine, Zagazig University where they are processed and diagnosed as breast carcinoma by routine H\&E histopathological examination pathologests from Department of Pathology, Faculty of Medicine, Benghazi University, Benghazi, Libya revise the diagnosis of all slides. Pathologists from Pathology Department, Faculty of Medicine, Zagazig University Zagazig, Egypt and from Department of Pathology, Faculty of Medicine, Benghazi University, Benghazi, Libya used the American Joint Committee on Cancer staging system classification ( $8^{\text {th }}$ edition) for cancer staging (Giuliano et al., 2017) and the Nottingham (Elston-Ellis) modification of the [Scarff. Bloom Richardson] grading system for cancer grading (Elston, Ellis IO, 2002). We identified age, tumor size, histopathological subtype, grade, stage of cancer by examination of the patient's and the slide files of the Pathology Department. ER, PR hormonal receptors\& Her2 neu expressions and Ki67 labeling index were evaluated for all cases. All cases are followed up for therapy response, recurrence and survival in clinical oncology and laboratory medicine department, faculty of medicine, Zagazig University. We followed up our patients until death or until the last seen alive with the median follow-up period of 30 month with range from (15-36 month).

The technique of immunohistochemical staining:-

We used the common technique of streptavidin-biotin immunoperoxidase for staining (Hsu et al., 1981). We cut sections of five- $\mu \mathrm{m}$ thichness of formalin-fixed, paraffin-embedded tissues blocks prepared from surgically excised breast carcinoma tissue; we placed sections on positively charged slides, de-wax and rehydrate them. We block the 
activity of endogenous peroxidase; we exposed the sections to heat for antigen retrieval in the autoclave, incubated them overnight with primary mouse monoclonal anti- HIF-1 $\alpha$ (Calbiochem, Germany, diluted 1:300), and primary rabbit polyclonal anti-CAIX (Santa Cruz Bioscience, Santa Cruz, CA, USA, diluted 1:100) antibodies at $4^{\circ} \mathrm{C}$. We used the chromogen diaminobenzidine substrate (DAB). Lastly we counterstained sections with hematoxylin. We included positive and negative controls of both markers in all cases. We considered sections from cervical squamous cell carcinoma that was positive for HIF-1 $\alpha$, and CAIX as positive control for both markers [Lee et al., 2008)]. And we have omitted the primary antibodies and replaced the by non-immune serum for the negative controls.

Evaluation of immunohistochemical expression of HIF- $1 \alpha$ and CAIX:-

We considered any dark stained nuclei and positive membranous \& cytoplasmic stain in $>1 \%$ of the tumor cells as positive for HIF-1 $\alpha$ and CAIX respectively [Trastour et al., 2007].

\section{Results:-}

Ninety females' patients were included in our study with 49 (54.4\%) patients were >55years. All detailed clinicopathological criteria are included in table (1).

\section{HIF-1 $\alpha$ expression, correlation to clinical and histopathological findings Tables 2 \&3; fig 1}

HIF-1 $\alpha$ positive expression in breast carcinoma was significantly correlated with older age of the patients, higher grade and advanced stage of the tumor. HIF-1 $\alpha$ positive expression was significantly correlated with aggressive molecular subtypes as HER2 amplified and triple negative subtypes, presence of lymph node metastases, high KI67 index $(\mathrm{p}<0.001$ for all of them), presence of distant metastasis $(\mathrm{p}=0.041)$, negative ER $(\mathrm{p}=0.005) \& \mathrm{PR}(\mathrm{p}=0.009)$, but it had no significant correlation with histopathological subtype of breast cancer.

CAIX expression, correlation to clinical and histopathological findings Tables 2 \&3; fig 2

The positive expression of CAIX in breast carcinoma was significantly correlated with older age of the patients, advanced stage of the tumor, aggressive molecular type, presence of lymph node metastases, high KI67 index, aggressive molecular subtypes as HER2 amplified and triple negative subtypes, $(\mathrm{p}<0.001$ for all of them), higher grade $(\mathrm{p}=0.02)$ negative ER $(\mathrm{p}=0.008) \& \mathrm{PR}(\mathrm{p}=0.027)$ hormonal receptors, But it had no significant correlation with histopathological subtype of breast cancer or presence of distant metastasis.

The expression of HIF- $1 \alpha$ and CAIX in breast carcinoma was significantly positively correlated with each other $(\mathrm{p}<0.001)$.

Survival analysis: Tables 4 \&5; fig 3

After the follow-up period of 30 months $27.8 \%$ of patients died; The 3 -year overall survival rate was $74.4 \%$ with a mean of $32.6 \pm 0.62$ months (95\% CI; 31.4 - 33.8 months) while the median OS was not detected.

The 3 -year RFS rate was $56.1 \%$ with a mean of $30.3 \pm 0.8$ months (95\% CI; 28.7- 32.2 months); however the median RFS was not detected

At the end of follow up there was 38.9\% of patients [35/90 patients] developed cancer recurrence.

In multi variant analysis LN metastasis is the most significant factor of RFS \& OS rates

\section{Progression follow-up and survival results in correlation to HIF- $1 \alpha$ \& CAIX expression}

Cases with positive HIF- $1 \alpha$ \& CAIX expression had a higher rate of carcinoma recurrence $(\mathrm{p}<0.001)$.

In univariant analysis patients with positive HIF-1 $\alpha$ \& CAIX expression had poor RFS and 3 year OS rates $(\mathrm{p}=0.007)$.

We found a significant correlations between HIF-1 $\alpha$ \& CAIX positive expressions in carcinoma of the breast $(\mathrm{p}<0.001)$. Table 4; Fig 3

Table1:- The clinicopathological features of our 90 patients.

\begin{tabular}{|l|l|l|}
\hline \multicolumn{2}{|l|}{ Clinicopathological feature } & No. (\%) \\
\hline \multirow{2}{*}{ Age group } & $<55 \mathrm{y}$ & \\
\cline { 2 - 3 } & $>55 \mathrm{y}$ & $41(45.6 \%)$ \\
\hline Pathology & IDC (NST) & $79(54.4 \%)$ \\
\hline
\end{tabular}




\begin{tabular}{|c|c|c|}
\hline & ILC & $20(22.2 \%)$ \\
\hline \multirow{3}{*}{ Grade } & 1 & $20(22.2 \%)$ \\
\hline & 2 & $40(44.4 \%)$ \\
\hline & 3 & $30(33.3 \%)$ \\
\hline \multirow[t]{2}{*}{ LVI } & Absent & $26(28.9 \%)$ \\
\hline & Present & $64(71.1 \%)$ \\
\hline \multirow[t]{2}{*}{ ER } & Negative & $42(46.7 \%)$ \\
\hline & Positive & $48(53.3 \%)$ \\
\hline \multirow[t]{2}{*}{$\mathbf{P R}$} & Negative & $42(46.7 \%)$ \\
\hline & Positive & $48(53.3 \%)$ \\
\hline \multirow[t]{4}{*}{ ER/PR } & Positive/Positive & $44(48.9 \%)$ \\
\hline & Positive/Negative & $4(4.4 \%)$ \\
\hline & Negative/Positive & $4(4.4 \%)$ \\
\hline & Negative/Negative & $38(42.2 \%)$ \\
\hline \multirow[t]{2}{*}{ HER2 } & Negative & $54(60.0 \%)$ \\
\hline & Positive & $36(40.0 \%)$ \\
\hline \multirow[t]{2}{*}{ KI 67} & Low & $31(34.4 \%)$ \\
\hline & High & $59(65.6 \%)$ \\
\hline \multirow[t]{4}{*}{ Molecular } & Luminal A & $34(37.8 \%)$ \\
\hline & Luminal B & $12(13.3 \%)$ \\
\hline & HER2 amplified & $24(26.7 \%)$ \\
\hline & Triple -ve & $20(22.2 \%)$ \\
\hline \multirow[t]{2}{*}{$\mathbf{L N}$} & Negative & $26(28.9 \%)$ \\
\hline & Positive & $64(71.1 \%)$ \\
\hline \multirow[t]{2}{*}{ DM } & Absent & $69(76.7 \%)$ \\
\hline & Present & $21(23.3 \%)$ \\
\hline \multirow[t]{4}{*}{ T classification } & $\mathrm{T} 1$ & $19(21.1 \%)$ \\
\hline & $\mathrm{T} 2$ & $37(41.1 \%)$ \\
\hline & T3 & $21(23.3 \%)$ \\
\hline & $\mathrm{T} 4$ & $13(14.4 \%)$ \\
\hline \multirow[t]{4}{*}{ N classification } & N0 & $26(28.9 \%)$ \\
\hline & N1 & $18(20.0 \%)$ \\
\hline & N2 & $27(30.0 \%)$ \\
\hline & N3 & $19(21.1 \%)$ \\
\hline \multirow[t]{4}{*}{ Stage } & Stage I & $14(15.6 \%)$ \\
\hline & Stage II & $30(33.3 \%)$ \\
\hline & Stage III & $25(27.8 \%)$ \\
\hline & Stage IV & $21(23.3 \%)$ \\
\hline
\end{tabular}

Table2:- Frequency of HIF-1a and CAIX expressions in our 90 patients.

\begin{tabular}{|c|c|c|}
\hline \multicolumn{2}{|l|}{ Markers } & No. $(\%)$ \\
\hline \multirow[t]{2}{*}{ CAIX } & Negative & $39(43.3 \%)$ \\
\hline & Positive & $51(56.7 \%)$ \\
\hline \multirow[t]{2}{*}{ 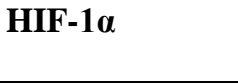 } & Negative & $32(35.6 \%)$ \\
\hline & Positive & $58(64.4 \%)$ \\
\hline \multirow[t]{3}{*}{ CAIX / HIF-1a } & Positive/Positive & $51(56.7 \%)$ \\
\hline & Negative/Positive & $7(7.8 \%)$ \\
\hline & Negative/Negative & $32(35.6 \%)$ \\
\hline
\end{tabular}

Table3:- Association of clinicopathological features with HIF-1a and CAIX expressions in our 90 patients.

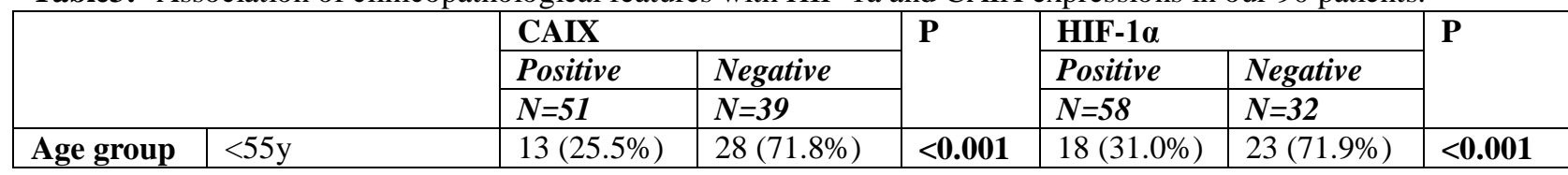




\begin{tabular}{|c|c|c|c|c|c|c|c|}
\hline & $>55 y$ & $38(74.5 \%)$ & $11(28.2 \%)$ & & $40(69.0 \%)$ & $9(28.1 \%)$ & \\
\hline \multirow[t]{2}{*}{ Pathology } & IDC (NST) & $40(78.4 \%)$ & $30(76.9 \%)$ & \multirow[t]{2}{*}{0.865} & $45(77.6 \%)$ & $25(78.1 \%)$ & \multirow[t]{2}{*}{0.953} \\
\hline & ILC & $11(21.6 \%)$ & $9(23.1 \%)$ & & $13(22.4 \%)$ & $7(21.9 \%)$ & \\
\hline \multirow[t]{3}{*}{ Grade } & 1 & $8(15.7 \%)$ & $12(30.8 \%)$ & \multirow[t]{3}{*}{0.02} & $9(15.5 \%)$ & $11(34.4 \%)$ & \multirow[t]{3}{*}{0.001} \\
\hline & 2 & $20(39.2 \%)$ & $20(51.3 \%)$ & & $22(37.9 \%)$ & $18(56.3 \%)$ & \\
\hline & 3 & $23(45.1 \%)$ & $7(17.9 \%)$ & & $27(46.6 \%)$ & $3(9.4 \%)$ & \\
\hline \multirow[t]{2}{*}{ LVI } & Absent & $5(9.8 \%)$ & $21(53.8 \%)$ & \multirow[t]{2}{*}{$<0.001$} & $5(8.6 \%)$ & $21(65.6 \%)$ & \multirow[t]{2}{*}{$<0.001$} \\
\hline & Present & $46(90.2 \%)$ & $18(46.2 \%)$ & & $53(91.4 \%)$ & $11(34.4 \%)$ & \\
\hline \multirow[t]{2}{*}{ ER } & Negative & $30(58.8 \%)$ & $12(30.8 \%)$ & \multirow[t]{2}{*}{0.008} & $34(58.6 \%)$ & $8(25.0 \%)$ & \multirow[t]{2}{*}{0.005} \\
\hline & Positive & $21(41.2 \%)$ & $27(69.2 \%)$ & & $24(41.4 \%)$ & $24(75.0 \%)$ & \\
\hline \multirow[t]{2}{*}{ PR } & Negative & $29(56.9 \%)$ & $13(33.3 \%)$ & \multirow[t]{2}{*}{$\mathbf{0 . 0 2 7}$} & $33(56.9 \%)$ & $9(28.1 \%)$ & \multirow[t]{2}{*}{0.009} \\
\hline & Positive & $22(43.1 \%)$ & $26(66.7 \%)$ & & $25(43.1 \%)$ & $23(71.9 \%)$ & \\
\hline \multirow[t]{4}{*}{ ER/PR } & Positive/Positive & $18(35.3 \%)$ & $26(66.7 \%)$ & \multirow[t]{4}{*}{0.017} & $21(36.2 \%)$ & $23(71.9 \%)$ & \multirow[t]{4}{*}{0.01} \\
\hline & Positive/Negative & $3(5.9 \%)$ & $1(2.6 \%)$ & & $3(5.2 \%)$ & $1(3.1 \%)$ & \\
\hline & Negative/Positive & $4(7.8 \%)$ & $0(0.0 \%)$ & & $4(6.9 \%)$ & $0(0.0 \%)$ & \\
\hline & Negative/Negative & $26(51.0 \%)$ & $12(30.8 \%)$ & & $30(51.7 \%)$ & $8(25.0 \%)$ & \\
\hline \multirow[t]{2}{*}{ HER2 } & Negative & $21(41.2 \%)$ & $33(84.6 \%)$ & \multirow[t]{2}{*}{$<0.001$} & $27(46.6 \%)$ & $27(84.4 \%)$ & \multirow[t]{2}{*}{$<0.001$} \\
\hline & Positive & $30(58.8 \%)$ & $6(15.4 \%)$ & & $31(53.4 \%)$ & $5(15.6 \%)$ & \\
\hline \multirow[t]{2}{*}{ KI 67} & Low & $8(15.7 \%)$ & $23(59.0 \%)$ & \multirow[t]{2}{*}{$<0.001$} & $10(17.2 \%)$ & $21(65.6 \%)$ & \multirow[t]{2}{*}{$<0.001$} \\
\hline & High & $43(84.3 \%)$ & $16(41.0 \%)$ & & $48(82.8 \%)$ & $11(34.4 \%)$ & \\
\hline Molecular & Luminal A & $11(21.6 \%)$ & $23(59.0 \%)$ & $<0.001$ & $13(22.4 \%)$ & $21(65.6 \%)$ & $<0.001$ \\
\hline & Luminal B & $9(17.6 \%)$ & $3(7.7 \%)$ & & $10(17.2 \%)$ & $2(6.3 \%)$ & \\
\hline & HER2 amplified & $21(41.2 \%)$ & $3(7.7 \%)$ & & $21(36.2 \%)$ & $3(9.4 \%)$ & \\
\hline & Triple -ve & $10(19.6 \%)$ & $10(25.6 \%)$ & & $14(24.1 \%)$ & $6(18.8 \%)$ & \\
\hline $\mathbf{L N}$ & Negative & $5(9.8 \%)$ & $21(53.8 \%)$ & $<0.001$ & $5(8.6 \%)$ & $21(65.6 \%)$ & $<0.001$ \\
\hline & Positive & $46(90.2 \%)$ & $18(46.2 \%)$ & & $53(91.4 \%)$ & $11(34.4 \%)$ & \\
\hline DM & Absent & $36(70.6 \%)$ & $33(84.6 \%)$ & 0.113 & $41(70.7 \%)$ & $28(87.5 \%)$ & 0.041 \\
\hline & Present & $15(29.4 \%)$ & $6(15.4 \%)$ & & $17(29.3 \%)$ & $4(12.5 \%)$ & \\
\hline $\mathbf{T}$ & $\mathrm{T} 1$ & $3(5.9 \%)$ & $16(41.0 \%)$ & $<0.001$ & $5(8.6 \%)$ & $14(43.8 \%)$ & $<0.001$ \\
\hline & $\mathrm{T} 2$ & $18(35.3 \%)$ & $19(48.7 \%)$ & & $22(37.9 \%)$ & $15(46.9 \%)$ & \\
\hline & T3 & $20(39.2 \%)$ & $1(2.6 \%)$ & & $21(36.2 \%)$ & $0(0.0 \%)$ & \\
\hline & $\mathrm{T} 4$ & $10(19.6 \%)$ & $3(7.7 \%)$ & & $10(17.2 \%)$ & $3(9.4 \%)$ & \\
\hline $\mathbf{N}$ & N0 & $5(9.8 \%)$ & $21(53.8 \%)$ & $<0.001$ & $5(8.6 \%)$ & $21(65.6 \%)$ & $<0.001$ \\
\hline & N1 & $9(17.6 \%)$ & $9(23.1 \%)$ & & $11(19.0 \%)$ & $7(21.9 \%)$ & \\
\hline & $\mathrm{N} 2$ & $21(41.2 \%)$ & $6(15.4 \%)$ & & $26(44.8 \%)$ & $1(3.1 \%)$ & \\
\hline & N3 & $16(31.4 \%)$ & $3(7.7 \%)$ & & $16(27.6 \%)$ & $3(9.4 \%)$ & \\
\hline Stage & Stage I & $0(0.0 \%)$ & $14(35.9 \%)$ & $<0.001$ & $0(0.0 \%)$ & $14(43.8 \%)$ & $<0.001$ \\
\hline & Stage II & $13(25.5 \%)$ & $17(43.6 \%)$ & & $16(27.6 \%)$ & $14(43.8 \%)$ & \\
\hline & Stage III & $23(45.1 \%)$ & $2(5.1 \%)$ & & $25(43.1 \%)$ & $0(0.0 \%)$ & \\
\hline & Stage IV & $15(29.4 \%)$ & $6(15.4 \%)$ & & $17(29.3 \%)$ & $4(12.5 \%)$ & \\
\hline HIF-1a & Negative & $0(0.0 \%)$ & $32(82.1 \%)$ & $<0.001$ & & & \\
\hline & Positive & $51(100.0 \%)$ & $7(17.9 \%)$ & & & & \\
\hline
\end{tabular}

Table4:- Univariate analysis of overall and Recurrence-Free Survival in relation to clinicopathological parameters of our 90 patients.

\begin{tabular}{|c|c|c|c|c|c|}
\hline \multicolumn{2}{|l|}{ Variables } & \multirow{3}{*}{$\begin{array}{l}\text { 3-year overall } \\
\text { survival Rate (\%) } \\
78.9 \% \\
54.5 \% \\
\end{array}$} & \multirow{3}{*}{$\begin{array}{l}\text { p-value } \\
0.001\end{array}$} & \multirow{2}{*}{$\begin{array}{l}\text { 3-year Recurrence } \\
\text { Free survival Rate } \\
(\%)\end{array}$} & \multirow{3}{*}{$\begin{array}{l}\text { p-value } \\
0.002\end{array}$} \\
\hline \multirow{2}{*}{ Age group } & $<55 \mathrm{y}$ & & & & \\
\hline & $>55 \mathrm{y}$ & & & $46.8 \%$ & \\
\hline \multirow[t]{2}{*}{ Pathology } & IDC (NST) & $67.6 \%$ & \multirow[t]{2}{*}{0.141} & $60.1 \%$ & \multirow{2}{*}{0.029} \\
\hline & ILC & $59.5 \%$ & & $39.7 \%$ & \\
\hline \multirow[t]{2}{*}{ Grade } & 1 & $100.0 \%$ & \multirow[t]{2}{*}{$<0.001$} & $90.0 \%$ & \multirow[t]{2}{*}{$<0.001$} \\
\hline & 2 & $45.7 \%$ & & $56.6 \%$ & \\
\hline
\end{tabular}




\begin{tabular}{|c|c|c|c|c|c|}
\hline & 3 & $47.5 \%$ & & $25.3 \%$ & \\
\hline \multirow[t]{2}{*}{ LVI } & Absent & $78.5 \%$ & \multirow[t]{2}{*}{0.032} & $80.2 \%$ & \multirow[t]{2}{*}{0.001} \\
\hline & Present & $59.4 \%$ & & $44.8 \%$ & \\
\hline \multirow[t]{2}{*}{ ER } & Negative & $54.3 \%$ & \multirow[t]{2}{*}{0.004} & $38.9 \%$ & \multirow[t]{2}{*}{$<0.001$} \\
\hline & Positive & $74.7 \%$ & & $70.9 \%$ & \\
\hline \multirow[t]{2}{*}{ PR } & Negative & $57.6 \%$ & \multirow[t]{2}{*}{0.010} & $40.7 \%$ & \multirow[t]{2}{*}{$<0.001$} \\
\hline & Positive & $74.3 \%$ & & $70.0 \%$ & \\
\hline \multirow[t]{4}{*}{ ER/PR } & Positive/Positive & $73.8 \%$ & \multirow[t]{4}{*}{0.016} & $68.3 \%$ & \multirow[t]{4}{*}{$<0.001$} \\
\hline & Positive/Negative & $100.0 \%$ & & $100.0 \%$ & \\
\hline & Negative/Positive & $100.0 \%$ & & $100.0 \%$ & \\
\hline & Negative/Negative & $52.2 \%$ & & $32.8 \%$ & \\
\hline \multirow[t]{2}{*}{ HER2 } & Negative & $70.2 \%$ & \multirow[t]{2}{*}{0.039} & $61.1 \%$ & \multirow[t]{2}{*}{0.027} \\
\hline & Positive & $60.7 \%$ & & $50.1 \%$ & \\
\hline \multirow[t]{2}{*}{ KI 67} & Low & $69.8 \%$ & \multirow[t]{2}{*}{0.135} & $63.5 \%$ & \multirow[t]{2}{*}{0.094} \\
\hline & High & $64.8 \%$ & & $54.4 \%$ & \\
\hline \multirow[t]{4}{*}{ Molecular } & Luminal A & $69.6 \%$ & \multirow[t]{4}{*}{$<0.001$} & $62.7 \%$ & \multirow[t]{4}{*}{$<0.001$} \\
\hline & Luminal B & $100.0 \%$ & & $100.0 \%$ & \\
\hline & HER2 amplified & $33.5 \%$ & & $20.8 \%$ & \\
\hline & Triple -ve & $71.6 \%$ & & $59.0 \%$ & \\
\hline \multirow[t]{2}{*}{$\mathbf{L N}$} & Negative & $78.5 \%$ & \multirow[t]{2}{*}{0.032} & $80.2 \%$ & \multirow[t]{2}{*}{0.001} \\
\hline & Positive & $59.4 \%$ & & $44.8 \%$ & \\
\hline \multirow[t]{2}{*}{ DM } & Absent & $73.9 \%$ & $<0.001$ & $69.9 \%$ & $<0.001$ \\
\hline & Present & $36.1 \%$ & & $0.0 \%$ & \\
\hline $\mathbf{T}$ & T1 & $88.9 \%$ & $<0.001$ & $83.3 \%$ & $<0.001$ \\
\hline & T2 & $70.1 \%$ & & $70.5 \%$ & \\
\hline & T3 & $45.4 \%$ & & $25.9 \%$ & \\
\hline & T4 & $35.2 \%$ & & $0.0 \%$ & \\
\hline $\mathbf{N}$ & N0 & $78.5 \%$ & $<0.001$ & $80.2 \%$ & $<0.001$ \\
\hline & N1 & $74.7 \%$ & & $68.8 \%$ & \\
\hline & $\mathrm{N} 2$ & $69.9 \%$ & & $44.1 \%$ & \\
\hline & N3 & $25.9 \%$ & & $23.7 \%$ & \\
\hline Stage & Stage I & $85.7 \%$ & $<0.001$ & $85.7 \%$ & $<0.001$ \\
\hline & Stage II & $72.8 \%$ & & $76.7 \%$ & \\
\hline & Stage III & $68.6 \%$ & & $44.8 \%$ & \\
\hline & Stage IV & $36.1 \%$ & & $0.0 \%$ & \\
\hline CAIX & Negative & $70.6 \%$ & 0.043 & $69.6 \%$ & 0.005 \\
\hline & Positive & $60.5 \%$ & & $45.5 \%$ & \\
\hline HIF-1a & Negative & $74.5 \%$ & $\mathbf{0 . 0 3 7}$ & $72.5 \%$ & 0.007 \\
\hline & Positive & $60.1 \%$ & & $46.2 \%$ & \\
\hline CAIX / HIF-1 $1 \alpha$ & Positive/Positive & $60.5 \%$ & 0.102 & $45.5 \%$ & 0.016 \\
\hline & Negative/Positive & $40.0 \%$ & & $40.0 \%$ & \\
\hline & Negative/Negative & $74.5 \%$ & & $72.5 \%$ & \\
\hline
\end{tabular}

$\mathrm{P}$ value $<0.05$ is significant.

Table5:- Multivariate analysis of overall and Recurrence-Free Survival in relation to clinicopathological parameters of our 90 patients.

\begin{tabular}{|l|l|l|l|l|}
\hline \multirow{2}{*}{ Variables } & 3 Years RFS & 3 Years OS & Sig \\
\cline { 2 - 5 } & HR $(\mathbf{9 5}$ \% CI) & Sig. & HR $(\mathbf{9 5} \%$ CI $)$ & 0.130 \\
\hline Age $>\mathbf{5 5 y}$ & $1.2(0.3-5.03)$ & 0.770 & $3.7(0.68-20.06)$ & 0.430 \\
\hline Pathology & $1.1(0.42-2.8)$ & 0.870 & $0.7(0.23-1.89)$ & $<\mathbf{0 . 0 0 1}$ \\
\hline Grade & $2.7(1.17-6.2)$ & $\mathbf{0 . 0 2 0}$ & $6.4(1.98-20.76)$ & $\mathbf{0 . 0 1 0}$ \\
\hline LVI & $0.1(0.01-1.15)$ & 0.060 & $0.01(0.001-0.33)$ & 0.120 \\
\hline ER & $2.1(0.03-154.62)$ & 0.740 & $34.6(0.39-3081.22)$ & 0.180 \\
\hline PR & $0.1(0.01-0.94)$ & $\mathbf{0 . 0 4 0}$ & $0.2(0.01-2.23)$ & \\
\hline
\end{tabular}




\begin{tabular}{|l|l|l|l|l|}
\hline HER2 & $0.3(0.06-1.37)$ & 0.120 & $0.2(0.03-1.07)$ & 0.060 \\
\hline KI67 & $0.1(0.01-0.85)$ & $\mathbf{0 . 0 4 0}$ & $0.1(0.01-2.62)$ & 0.180 \\
\hline Molecular & $0.7(0.14-3.81)$ & 0.710 & $1.2(0.28-5.39)$ & 0.790 \\
\hline DM & $2.3(0.29-17.67)$ & 0.430 & $2.0(0.2-19.1)$ & 0.570 \\
\hline T & $2.1(0.74-6.04)$ & 0.160 & $1.7(0.55-5.41)$ & 0.350 \\
\hline N & $10.6(2.32-47.88)$ & $<\mathbf{0 . 0 0 1}$ & $56.3(5.52-574.02)$ & $<\mathbf{0 . 0 0 1}$ \\
\hline Stage & $0.4(0.07-2.69)$ & 0.360 & $0.1(0.02-1.11)$ & 0.060 \\
\hline CAIX & $0.9(0.17-5.01)$ & 0.920 & $0.3(0.05-2.17)$ & 0.240 \\
\hline HIF1a & $3.1(0.47-20.54)$ & 0.240 & $6.5(0.65-65.63)$ & 0.110 \\
\hline
\end{tabular}

HR: Hazard ratio; 95\%CI: 95\% confidence interval, $\mathrm{p}<0.05$ is significant. OS overall survival, RFS Recurrence free survival

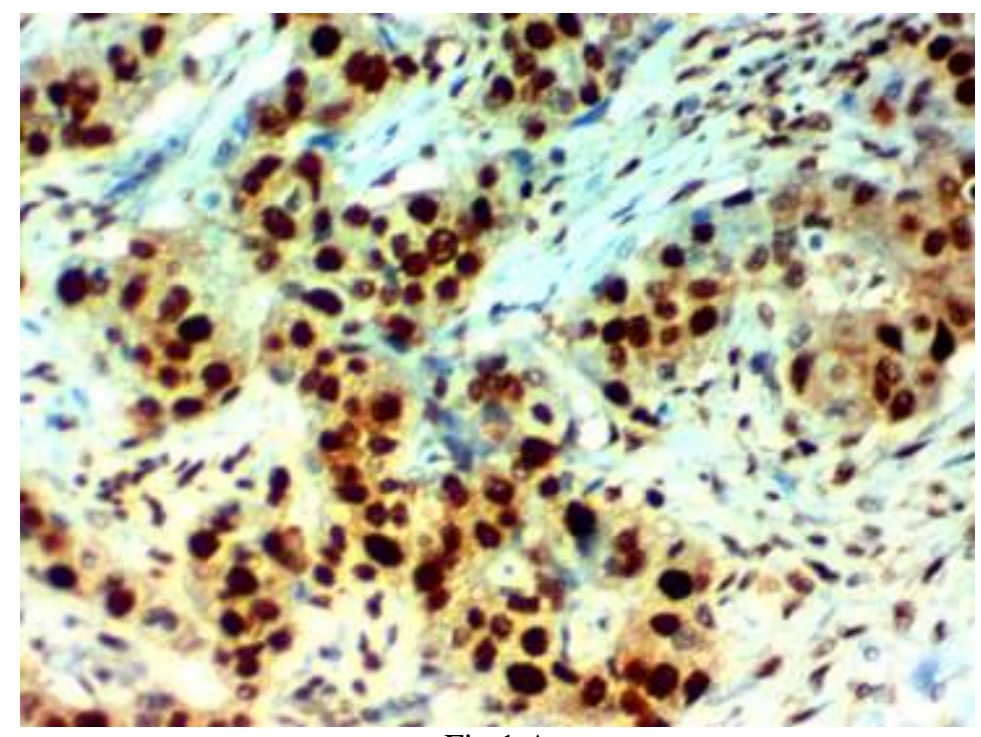

Fig $1 \mathrm{~A}$

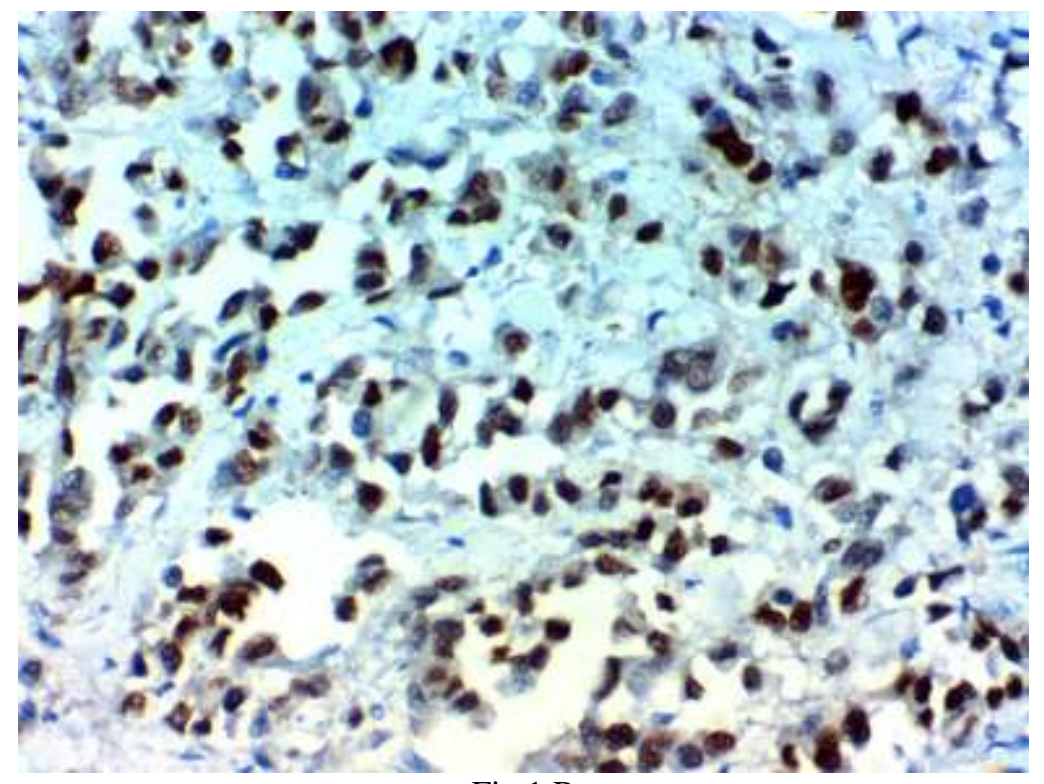

Fig $1 \mathrm{~B}$ 


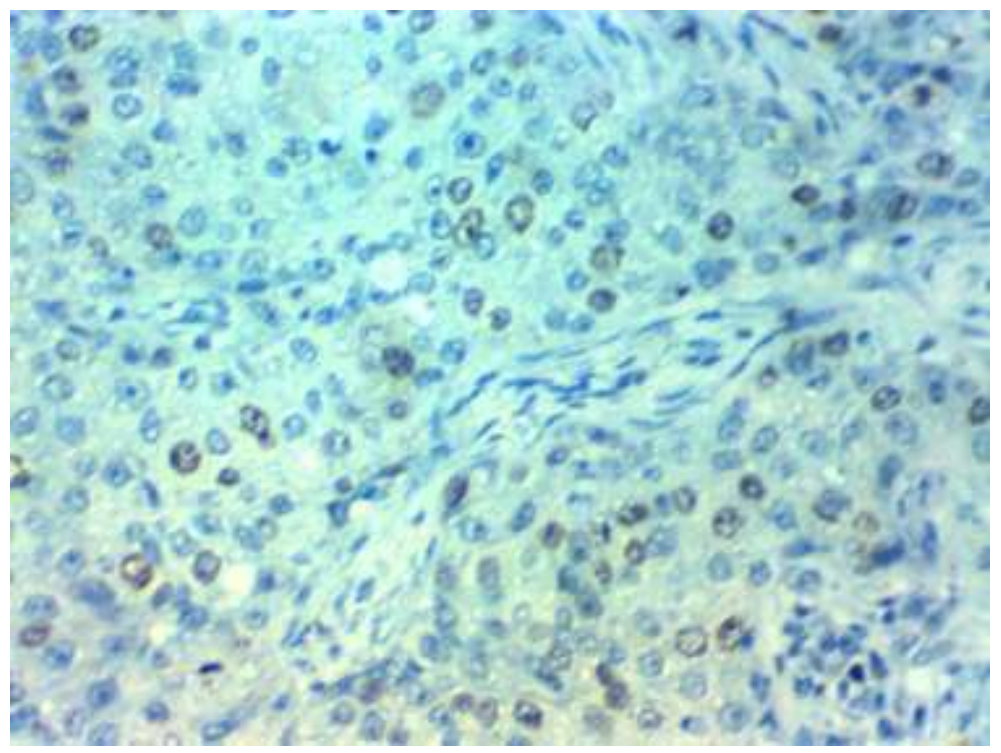

Fig $1 \mathrm{C}$

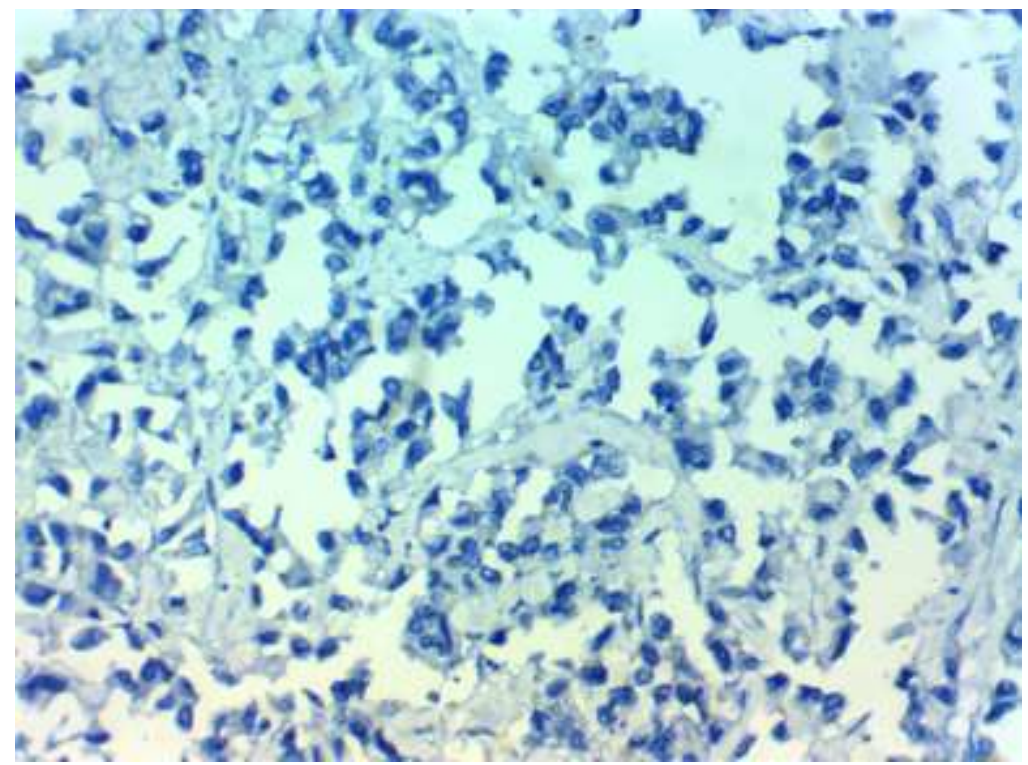

Fig $1 \mathrm{D}$

Figure1:- Immunohistochemical staining of HIF-1 $\alpha$ in invasive carcinoma of the breast: (A) High expression in the nucleus of high grade invasive duct carcinoma of the breast (NOS) x400. (B) High expression in the nucleus of high grade invasive lobular carcinoma of the breast $\mathrm{x} 400$ (C) Low expression in the nucleus of high grade invasive duct carcinoma of the breast (NOS) $\times 400$ (D) Low expression in the nucleus of high grade invasive lobular carcinoma of the breastx 1400 


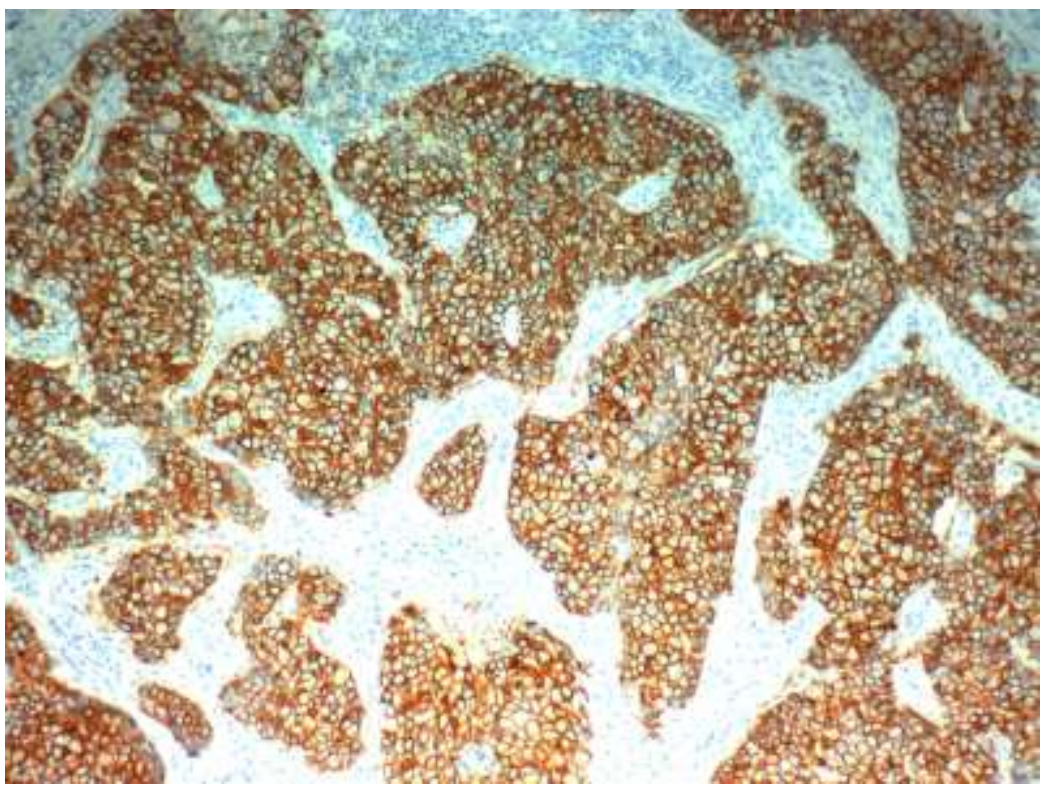

Fig $2 \mathrm{~A}$

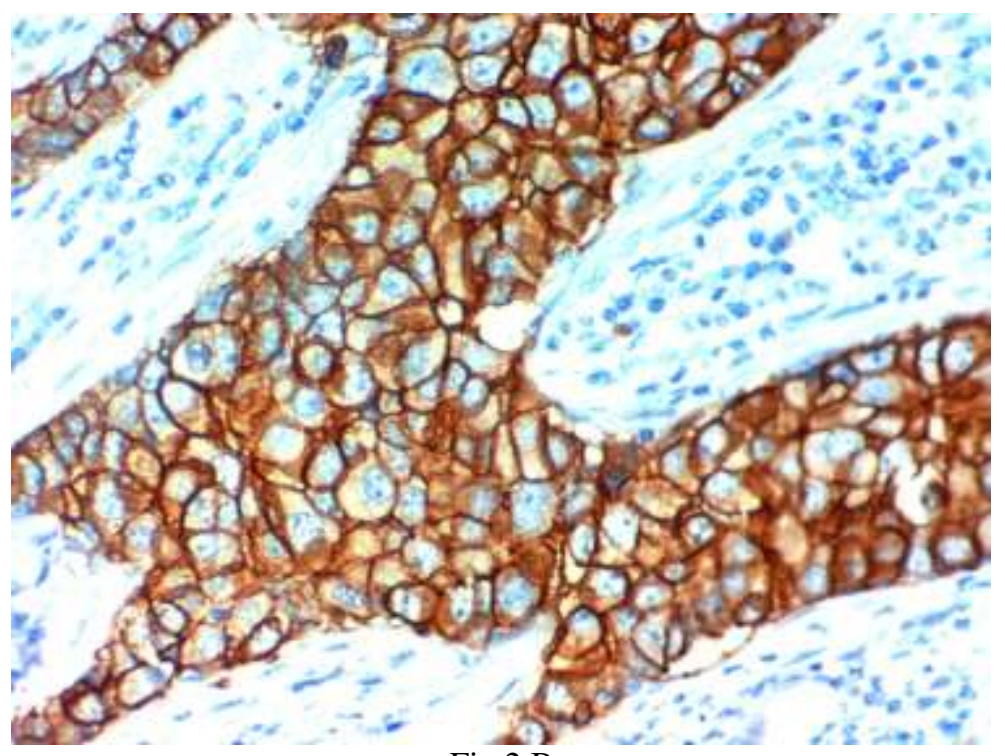

Fig 2 B 

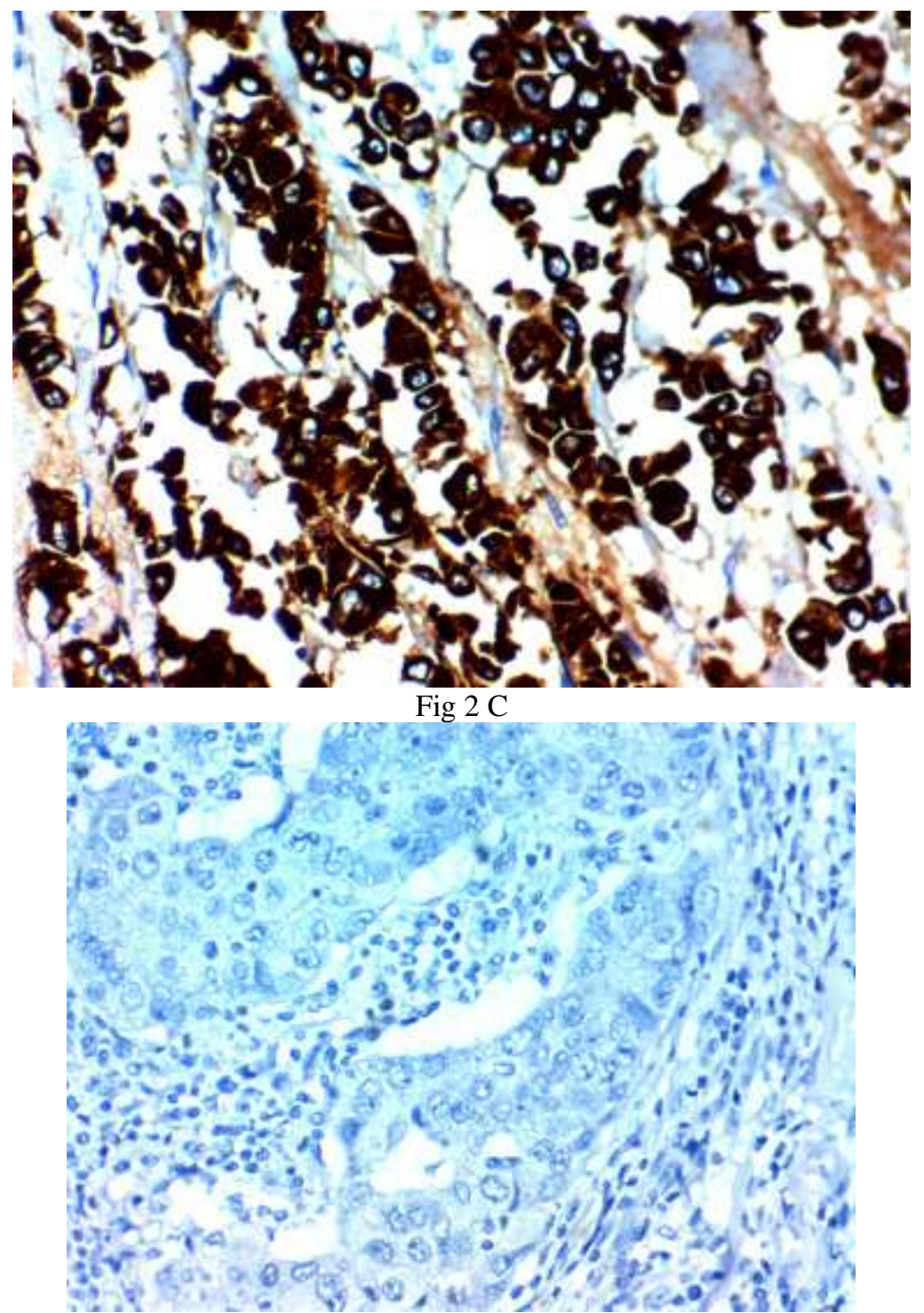

Fig $2 \mathrm{D}$

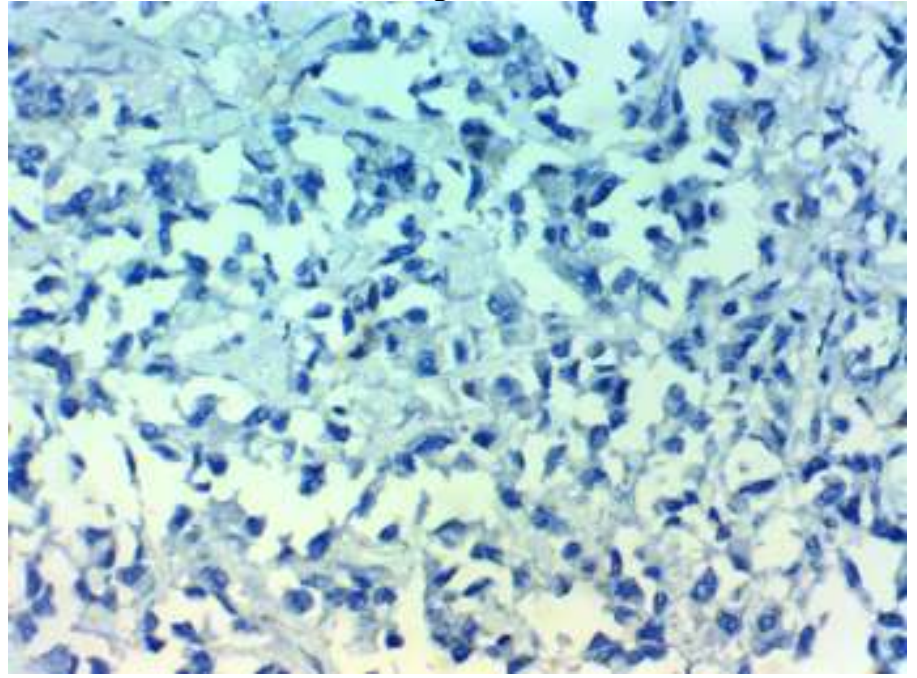

Fig 2 E 
Figure2:- Immunohistochemical staining of CAIX in invasive carcinoma of the breast: (A) High expression in the cytoplasm of high grade invasive duct carcinoma of the breast (NOS) x100. (B) High expression in the cytoplasm of high grade invasive duct carcinoma of the breast (NOS) $\mathrm{x} 400$. (C) High expression in the cytoplasm of high grade invasive lobular carcinoma of the breast $\mathrm{x} 400$ (D) Low expression in the cytoplasm of high grade invasive duct carcinoma of the breast (NOS)x400(E) Low expression in the cytoplasm of high grade invasive lobular carcinoma of the breastx 400

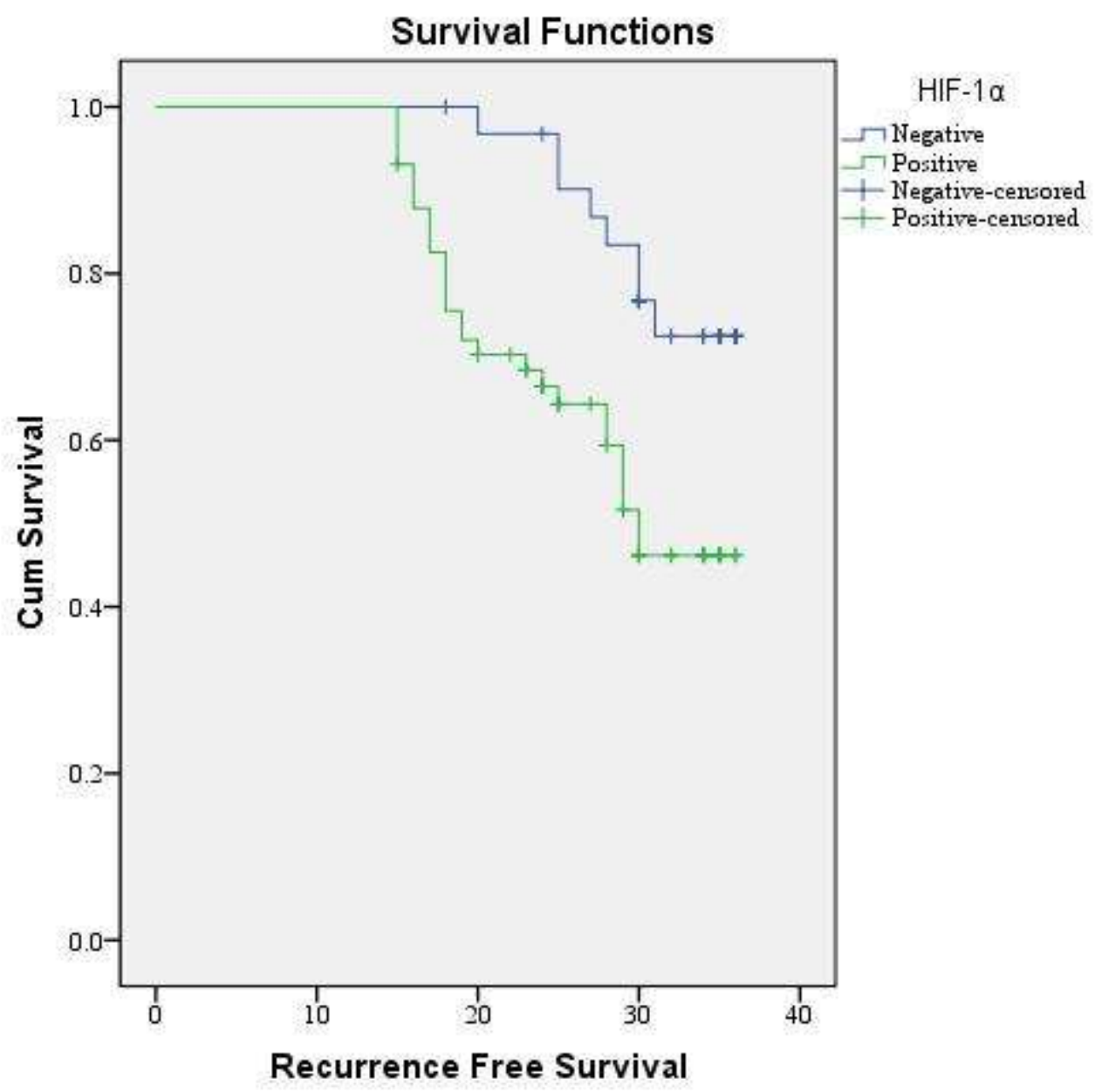

Fig 3 A 


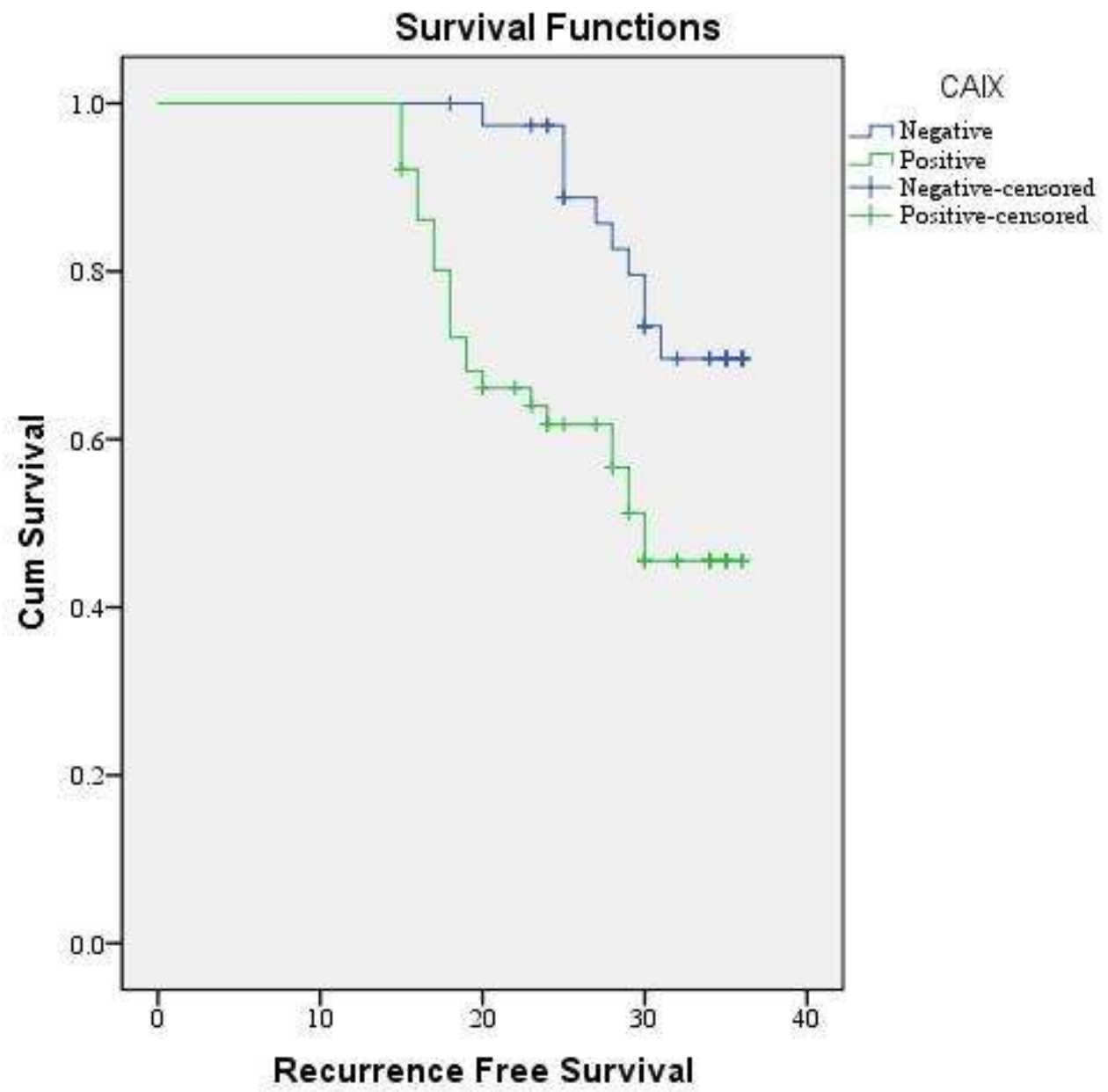

Fig 3 B 


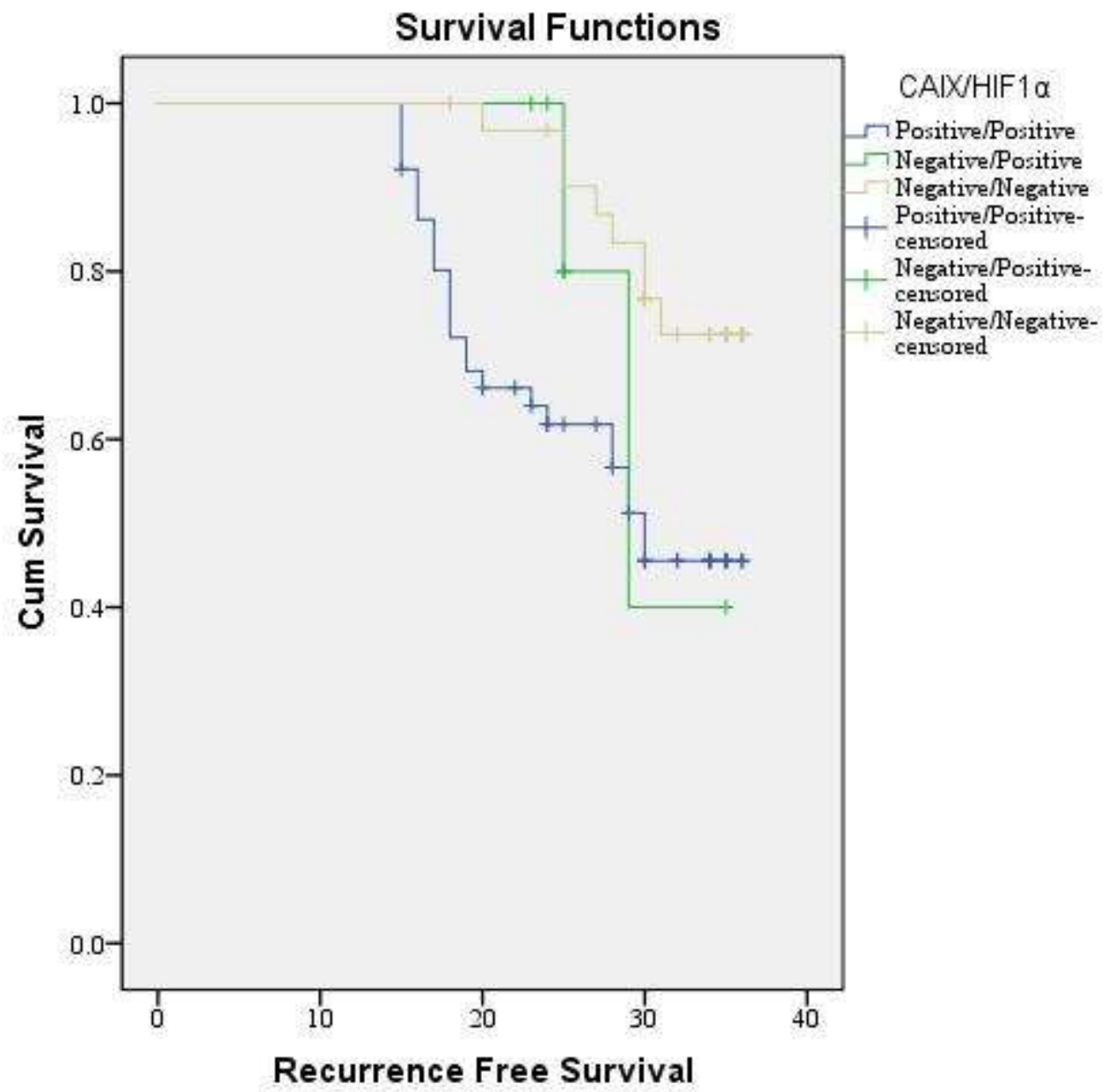

Fig $3 \mathrm{C}$ 


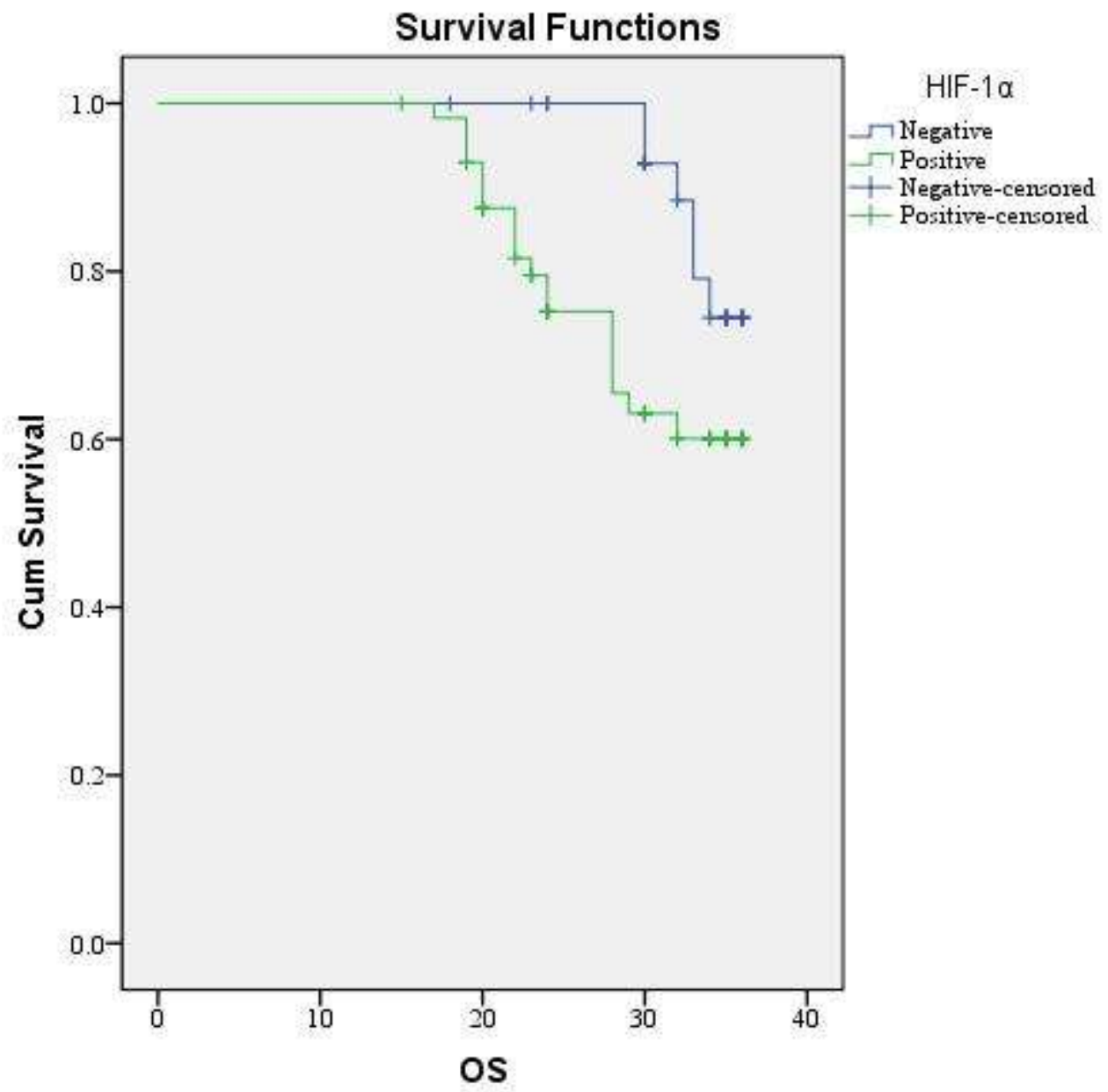

Fig 3 D 


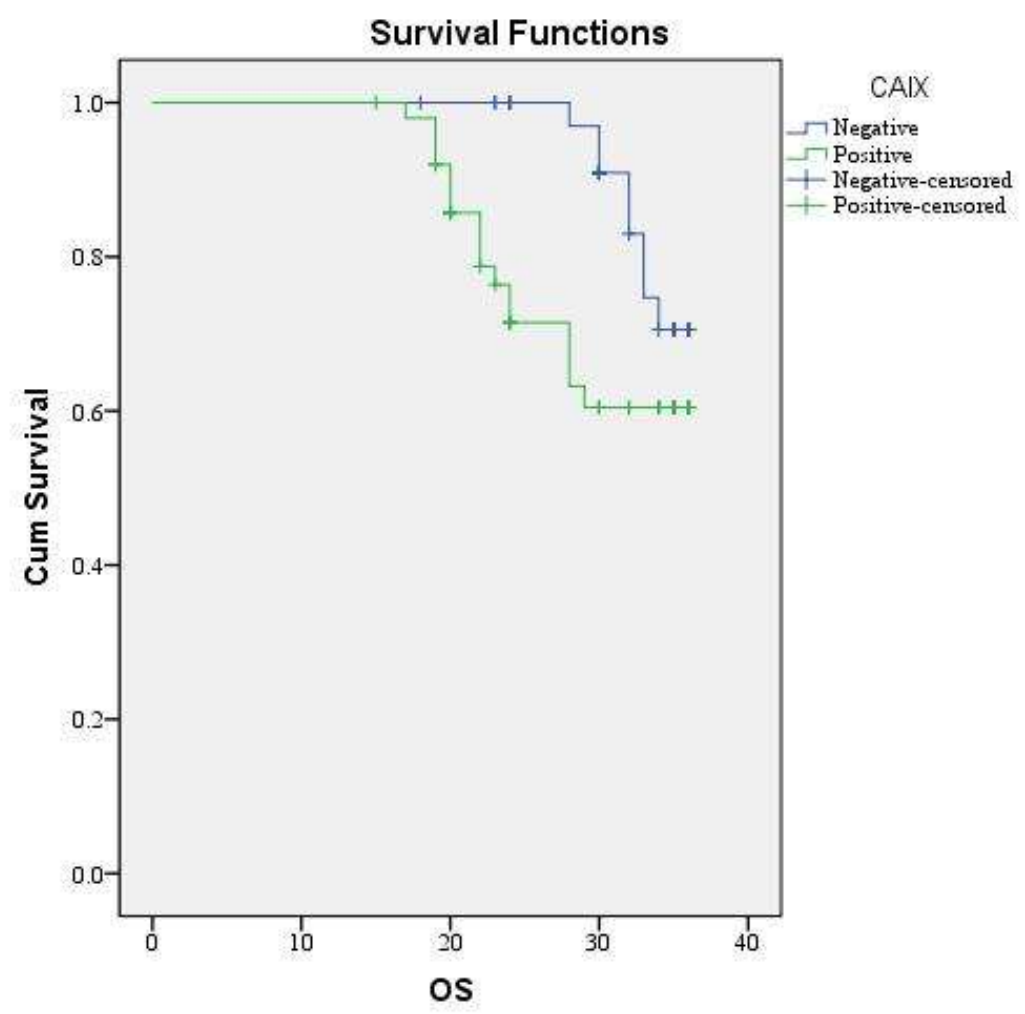

Fig 3 E

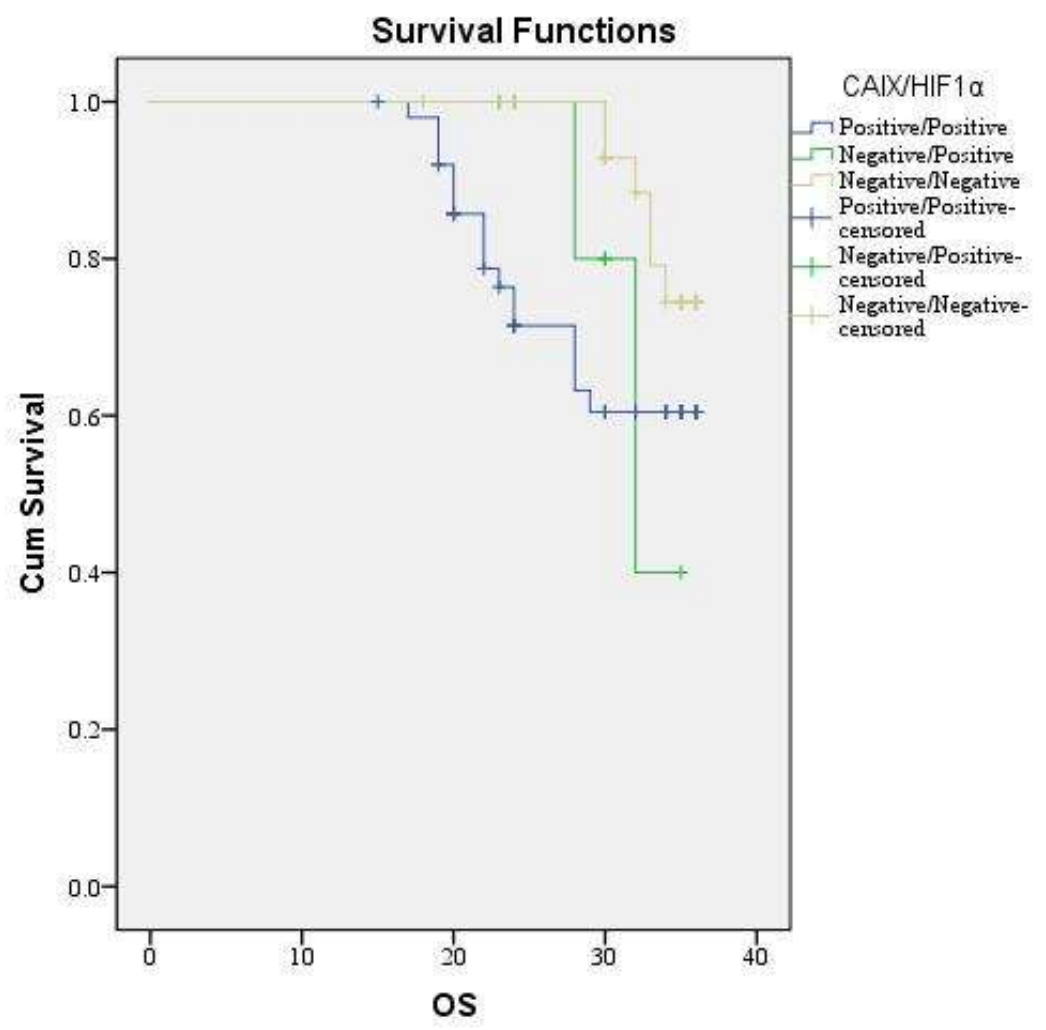

FIG $3 \mathrm{~F}$ 
Figure 3:- A.The 3-year Recurrence-Free survival Rate in relation to HIF-1 $\alpha$ Expression, B. The 3-year Recurrence-Free survival Rate in relation to CAIX Expression, C; The 3-year Recurrence-Free survival Rate in relation to CAIX/ HIF-1 $\alpha$ Expression, D; The 3-year overall survival in relation to HIF-1 $\alpha$ Expression, E; The 3year overall survival in relation to CAIX Expression, F. The 3-year overall survival in relation to CAIX / HIF-1 $\alpha$ Expression

\section{Discussion:-}

Former researchers had explored the role of HIF- $1 \alpha$ is involved in breast carcinogenesis [Kronblad et al., 2006], and detected that it could influence its growth rate and metastatic ability and subsequently could be associated with poor patient prognosis [Liu et al., 2015], but proved results are still conflicting and lacking accurate sharp data.

Our present results detected that when HIF- $1 \alpha$ positively expressed in breast carcinoma that will be significantly related to worse clinic pathological findings like older age of the patients, higher grade and advanced stage of the tumor, aggressive molecular subtypes, presence of LN and distant metastasis, also we found that cases with positive HIF-1 $\alpha$ expression had a higher rate of carcinoma recurrence, poor RFS and 3 year OS rates.

Our results were near results of former research that was done by Nalwoga et al., 2016 that evaluate the expression of HIF- $1 \alpha$ in relation to markers of angiogenesis and other clinicopathological criteria in a cohort of breast cancer from Africa and they detected that positive HIF-1 $\alpha$ expression was associated with increased tumor angiogenesis, high cancer cell proliferation rate that was evidenced by increased Ki-67 labeling index, high cancer grade that points to HIF- $1 \alpha$ as a poor prognostic marker of breast carcinoma and a therapeutic target for breast cancer patients.

We detected an association between HIF-1 $\alpha$ expression and the presence of LN \& distant metastasis in breast carcinoma; our results were near results of

Liu, et al., 2015 who had proved that proved that HIF- $1 \alpha$ is a regulator of cell hypoxia response and focused on HIF- $1 \alpha$ role in increasing breast carcinoma metastasis, as they explored that HIF-1 had several roles in metastasis, e.g. increasing malignant cells invasion, up-regulating epithelial-mesenchymal transition (EMT), and formation of metastatic niche. Liu, et al., 2015 also discuss the values of therapeutic benefits of targeting the HIF- $1 \alpha$ for management of breast cancer patients that is considered a recent therapeutic approach that could be used in combination with currently used therapies.

Wigerup et al., 2016 found that positive HIF-1 $\alpha$ protein expression is present in malignant tumors of many organs and that is associated with poor prognosis of carcinoma of cervix, endometrium and ovary. They stated that HIFs had many roles in cancer cells growth, proliferation, differentiation, angiogenesis, cancer cell metabolism, local invasion, lymph nodes and distant metastasis. Subsequently, HIFs could be responsible for to chemo- and radiotherapy resistance, so they are associated with poor prognosis of cancer patients.

In addition HIF- $1 \alpha$ could increase the expression of PD-L1 that is an immune checkpoint protein, which could be responsible for immune suppression (Noman et al., 2014).

But Wigerup et al., 2016 stated that the prognostic value of HIF-1 $\alpha$ in breast carcinoma patients has conflicting results in many follow-up studies,

Zhang $\boldsymbol{e t}$ al. (2012) showed results similar to ours that there is strong association between HIF-1 $\alpha$ expression and presence of distant metastases in breast carcinoma, and also HIF- $1 \alpha$ increased the extravasation of breast carcinoma cells in the lung, that was explained by Wong et al., 2012 by the ability of HIF-1 in regulation of metastatic niche formation at distant sites before cancer cell arrival.

Solid malignant tumors, like carcinoma of the breast contain hypoxic areas due to presence of vascularization defects in these rapidly growing cancer cells. HIF- $1 \alpha$ plays an essential role in cancer cells adaptation to hypoxia by increasing transcription of many genes that could regulate angiogenesis, proliferation, invasion, and metastasis (Semenza, 2012).

Another mechanism by which HIF- $1 \alpha$ can act is by up-regulating epithelial-mesenchymal transition (EMT) process which is essential for tumor progression. EMT could be stimulated by hypoxia, by many mechanisms like HIF-1 $\alpha$ pathways in several human malignancies. HIF- $1 \alpha$ induce EMT by up-regulation of EMT transcription factors e.g. 
Twist, Snail, Slug and Zeb in many cancer types [Zhang et al., 2015], In addition, hypoxia is an angiogenesis stimulating agent via production of many HIF-1 transcription factors, moreover during the EMT process HIF- $1 \alpha$ stimulated angiogenesis by up-regulating VEGF transcription, and associated with microvessel growth which is an evidence of activated angiogenesis [Wigerup et al., 2016].

As we found that increased positive HIF- $1 \alpha$ expression shows strong association with poor outcome and dismal survival rates of breast carcinoma patients, so that hypoxia is considered a hallmark of aggressive behavior of many solid tumors and responsible for metastases and therapy resistance, so it is considered a cancer attractive therapeutic targets like the recently discovered HIF- $1 \alpha$ inhibitors Liu, et al., 2015.

Targeting hypoxic cancer cells have been explored by many approaches e.g. hypoxia-activated prodrugs, and HIF$1 \alpha$ specific targeting (Semenza, 2012).

HIF- $1 \alpha$ inhibitors, like digoxin and acriflavine, had potential therapeutic roles in decreasing cancer growth, invasion, metastasis and vascularization in breast cancer (Wong $\boldsymbol{e t}$ al., 2012), HIF- $1 \alpha$ targeting is considered as a novel therapeutic modality for management of breast cancer patients and improving their prognosis which could be used in combination with currently used therapies.

Many researchers have studied CAIX expression in a plethora of human malignancies and stated that it was associated with poor patient's outcome, but its role in breast carcinoma patients still needs further clarifications [Thiry et al., 2006].

Here we proved that positive CAIX expression in breast carcinoma tissues was correlated related to worse clinic pathological findings like older age of the patients, higher grade and advanced stage of the tumor, aggressive molecular subtypes, presence of LN metastasis, also we found that cases with positive CAIX expression had a higher rate of carcinoma recurrence, poor RFS and 3 year OS rates.

Glaberman et al., 2016 also found nearly the same, that CAIX expression was related to aggressive pathological phenotype, chemotherapy resistance and poor prognosis of patients with breast cancer.

We proved that the positive expression of CAIX in breast carcinoma was related to the presence of LN metastases, that was like results of Aomatsu et al., 2014] who proved the same results, and results of Keun-Yong et al., 2016 who found that positive CAIX expression was strongly correlated with sentinel LN metastasis in addition to invasion of lymphatic vessels by the primary tumors, also many previous studies proved results similar to us; Aomatsu, et al., 2014 found that breast cancer patients with positive expression of CAIX had lower pathologic complete response (pCR) rates when treated with neoadjuvant chemo-therapy.

We proved that the positive expression of CAIX in breast carcinoma was correlated to larger tumor size, higher tumor grade, stage and aggressive molecular type similar to our results Sch"utze et al. who detected upregulation of CAIX expression in breast carcinoma patients with advanced stages [Sch"utze et al., 2013]. And results of, Lou, et al., 2011, Chen et al., 2010 who found a positive correlation between CAIX expression, aggressive phenotype of breast carcinoma and poor patient prognosis.

In our study we found that patients with high CAIX expression had shorter RFS and 3 year OS rates. Similar to our results Tan et al., 2009, observed that that positive CAIX expression was related to chemo-resistance and shorter survival rates in breast cancer patients.

Thus, these data suggest that CAIX is a predictive and a prognostic marker for breast cancer patients.

Different from our results, Chen et al 2010 found no association between CAIX expression in breast cancer tissues and patients' nodal status tha could be explained by different number of patients, variable technique of staining and different antibody clone which gives different results.

There are multiple theories which could explain the association between positive CAIX expression and poor patients' outcome in breast cancer. That, CAIX expression is linked to cancer tissue hypoxia and acidosis and its upregulation is a step in cancer cells adaptation to survive under hypoxic conditions [Chen et al., 2010,], also, 
CAIX is related to cancer hypoxia and stimulates cancer cell spread and invasion, which incriminated tumor hypoxia to increase cancer cells invasion and metastasis [Shin et al., 2011]. Tumors with upregulation of CAIX could be able to maintain their intracellular $\mathrm{pH}$, but it increased acidification in extracellular space, which leads to extracellular matrix breakdown which could increase malignant cells invasive ability [Chen et al., 2010,, M"uller et al., 2011], in addition increased hypoxia in the malignant cells leads to genome instability. Also, CAIX could influence breast cancer stem cells growth and survival under hypoxic conditions [Lock et al., 2013].

That association of CAIX positive expression with aggressive clinicopathological and prognostic parameters of breast cancer patients that proved by our results and results of previous studies, could support the theory that discovering selective CAIX inhibitors could be used to manage cancer patients and improving their prognosis, moreover some of such inhibitors are in the preclinical setting and still under evaluation [Ward, et al., 2013, McDonald, et al., 2012].

We found positive correlation between HIF-1 $\alpha$ \& CAIX expression in breast cancer tissue, that was similar to results of Brennan et al., 2006, but different from Chen et al 2010, Tan et al., 2009, who found no an association between both markers expression, which could be explained by that they have done their studies on tissue microarray that allow analyses of results based on only minute tissue samples and their tissue sections were acquired from only non-necrotic areas.

In addition, Tan, et al., 2009 explained the absence of association between both markers expression in their study by different half-lives of HIF- $1 \alpha$ and CAIX, as HIF- $1 \alpha$ was found to be rapidly destroyed and removed within minutes of re-oxygenation (Jiang et al, 1996), while, CAIX has a longer half-life of 2-3 days [Rafajova et al, 2004), so they stated that they could not be present together.

Sobhanifar et al., (2005 found positive correlation between HIF-1 $\alpha$ and CAIX expression in breast carcinoma tissue, which was similar to ours but they detected CAIX expression only without HIF- $1 \alpha$ expression in perinecrotic regions in cancers, which is also due to differences in half-lives of HIF-1 $\alpha$ and CAIX [Tan et al., (2009].

\section{Summary, Conclusions and future suggestions:-}

HIF-1 and its downstream target HIF- $1 \alpha$ are considered regulators of cancer cell response to hypoxic stress and play important roles in breast carcinoma cells growth, invasion and metastasis.

Both markers, mainly HIF- $1 \alpha$, is involved in the key step of the metastatic process e.g. EMT, malignant cell invasion, and metastatic niche formation.

As we demonstrated that breast carcinogenesis is stimulated by cells adaptation to hypoxia and acidosis, moreover the glycolytic, acid-resistant phenotype that has HIF- $1 \alpha$ and CAIX positive expression is an aggressive phenotype.

Hence it will be better that tumor management strategies should aim at antagonizing the sequence of hypoxia, glycolysis and acidosis.

Moreover, identification of the metabolic phenotype of breast carcinoma will allow discovering to novel therapeutic modalities.

Also, the aggressive triple negative molecular subtype that is difficult to treat, as they are both chemo-resistant and hormonal non-responsive, and as we detected that such subtype showed positive expression of both HIF- $1 \alpha$ and its downstream target CAIX, so targeting them both e.g. targeting HIF- $1 \alpha$ with its inhibitors, gene therapies and CAIX inhibitors could be of particular importance in managing this aggressive cancer and improving patients prognosis (Supuran, 2008). The combination of HIF- $1 \alpha$ \& CAIX inhibitors with existing therapeutic modalities might be found to be useful clinically.

Clinical therapeutic trials are needed to determine if they could increase the survival of patients having breast cancer alone or in addition to currently used therapies.

Future studies are needed to discover more specific HIF- $1 \alpha$ \& CAIX inhibitors, to study their detailed mechanism of action, and to include them in clinical therapeutic trials of breast cancer patients. 


\section{References:-}

1. Aomatsu N, Yashiro M, Kashiwagi S, Kawajiri H, Takashima T, Ohsawa M, et al. Carbonic anhydrase 9 is associated with chemosensitivity and prognosis in breast cancer patients treated with taxane and anthracycline. BMC Cancer. 2014; 14:400.

2. Brennan DJ, Jirstrom K, Kronblad A, Millikan RC, Landberg G, Duffy MJ, Ryden L, Gallagher WM, O'Brien SL. CA IX is an independent prognostic marker in premenopausal breast cancer patients with one to three positive lymph nodes and a putative marker of radiation resistance. Clin Cancer Res 2006;12:6421-6431

3. Bussink J, Kaanders JH, van der Kogel AJ. Tumor hypoxia at the micro-regional level: clinical relevance and predictive value of exogenous and endogenous hypoxic cell markers. Radiother Oncol. 2003; 67:3-15. [PubMed: 12758235].

4. C. Ward, S. P. Langdon, P. Mullen et al., "New strategies for targeting the hypoxic tumour microenvironment in breast cancer," Cancer Treatment Reviews, 2013; 39:171-179.

5. C.-L. Chen, J.-S. Chu, W.-C. Su, S.-C. Huang, and W.-Y. Lee, "Hypoxia and metabolic phenotypes during breast carcinogenesis: expression of HIF-1alpha, GLUT1, and CAIX,"Virchows Archiv, 2010; 457: 53-61,.

6. D. Sch"utze, K. Milde-Langosch, I. Witzel et al., "Relevance of cellular and serum carbonic anhydrase IX in primary breast cancer," Journal of Cancer Research and Clinical Oncology, 2013; 139: 747-754,.

7. E. Y. Tan, M. Yan, L. Campo et al., "The key hypoxia regulated gene CAIX is upregulated in basal-like breast tumours and is associated with resistance to chemotherapy," British Journal of Cancer, 2009;100:405-411,.

8. Elston CW, Ellis IO. Pathological prognostic factors in breast cancer I. The value of histological grade in breast cancer: experience from a large study with long-term follow-up. Histopathology 2002; 19:403-410.

9. F. E. Lock, P. C. McDonald, Y. Lou et al., "Targeting carbonic anhydrase IX depletes breast cancer stem cells within the hypoxic niche," Oncogene, 2013;32: 5210-5219,

10. Giuliano AE1, Connolly JL2, Edge SB3, Mittendorf EA4, Rugo HS5, Solin LJ6, et al., Breast Cancer-Major changes in the American Joint Committee on Cancer eighth edition cancer staging manual. CA Cancer J Clin. 2017 Jul 8;67(4):290-303. doi: 10.3322/caac.21393.

11. Glaberman UB, Marron M, Chalasani P, Livingston R, Iannone M, Specht J et al., Circulating Carbonic Anhydrase IX and Antiangiogenic Therapy in Breast Cancer Disease Markers Volume 2016, Article ID 9810383, 7 pages http://dx.doi.org/10.1155/2016/9810383

12. Hsu SM, Raine L and Fanger H. Use of avidin-biotin-peroxidase complex (ABC) in immunoperoxidase techniques: a comparison between $\mathrm{ABC}$ and unlabeled antibody (PAP) procedures. J Histochem Cytochem. $1981 ; 29: 577-580$.

13. Jiang BH, Semenza GL, Bauer C, Marti HH Hypoxia-inducible factor 1 levels vary exponentially over a physiologically relevant range of O2 tension. Am J Physiol (1996) ; 271: C1172-C1180

14. Keun-Yong Eom, Min Hye Jang, So Yeon Park, Eun Young Kang, Sung Won Kim, Jee Hyun Kim, Jae-Sung Kim, In Ah Kim, The Expression of Carbonic Anhydrase (CA) IX/XII and Lymph Node Metastasis in Early Breast Cancer Cancer Res Treat. 2016;48(1):125-132.

15. Kronblad A, Jirstrom K, Ryden L, Nordenskjold B, Landberg G. Hypoxia inducible factor-1alpha is a prognostic marker in premenopausal patients with intermediate to highly differentiated breast cancer but not a predictive marker for tamoxifen response. Int J Cancer. 2006; 118(10):2609-2616. Epub 2005/12/29. doi: 10.1002/ijc.21676 PMID: 16381002

16. Lakhani SR, Ellis IO, Schnitt SJ, Tan PH and Van de Vijver MJ, eds. WHO Classification of Tumours of the Breast. Fourth ed. IARC, Lyon; 2012. ISBN.13.

17. Lee WY, Huang SC, Hsu KF, Tzeng CC, Shen WL . Roles for hypoxia-regulated genes during cervical carcinogenesis: somatic evolution during the hypoxia-glycolysis-acidosis sequence. Gynecol Oncol 2008; 108:377-384

18. Liu Z-j Semenza GL, Zhang H-f. Hypoxia-inducible factor 1 and breast cancer metastasis. J Zhejiang Univ Sci B. 2015; 16(1):32-43. doi: 10.1631/jzus.B1400221 PMID: PMC4288942

19. Nalwoga H, Ahmed L, Arnes JB, Wabinga H, Akslen LA. Strong Expression of Hypoxia-Inducible Factor-1 $\alpha$ (HIF-1 $\alpha$ ) Is Associated with Axl Expression and Features of Aggressive Tumors in African Breast Cancer PLOS ONE | DOI:10.1371/journal.pone.0146823 January 13, 2016

20. Noman, M. Z., Desantis, G., Janji, B., Hasmim, M., Karray, S., Dessen, P et al., PD-L1 is a novel direct target of HIF-1alpha, and its blockade under hypoxia enhanced MDSC-mediated T cell activation. J Exp Med 2014; 211: 781-790.

21. P. C. McDonald, J.-Y. Winum, C. T. Supuran, and S. Dedhar, "Recent developments in targeting carbonic anhydrase IX for cancer therapeutics," Oncotarget, , 2012; 3: 84-97. 
22. Rafajova M, Zatovicova M, Kettmann R, Pastorek J, Pastorekova S Induction by hypoxia combined with low glucose or low bicarbonate and high posttranslational stability upon reoxygenation contribute to carbonic anhydrase IX expression in cancer cells. Int J Oncol 2004;24: 995-1004.

23. Semenza GL. Defining the role of hypoxia-inducible factor 1 in cancer biology and therapeutics. Oncogene. 2010; 29:625-634. [PubMed: 19946328]

24. Semenza, G. L. Hypoxia-inducible factors: mediators of cancer progression and targets for cancer therapy. Trends Pharmacol Sci 2012; 33, 207-214.

25. Sobhanifar S, Aquino-Parsons C, Stanbridge EJ, Olive P. Reduced expression of hypoxia-inducible factor1alpha in perinecrotic regions of solid tumors. Cancer Res 2005; 65:7259-7266

26. Sugie T, Sawada T, Tagaya N, Kinoshita T, Yamagami K, Suwa H, et al. Comparison of the indocyanine green fluorescence and blue dye methods in detection of sentinel lymph nodes in early-stage breast cancer. Ann Surg Oncol. 2013; 20:2213-8.

27. Sun JD, Liu Q, Wang J, et al. Selective tumor hypoxia targeting by hypoxia-activated prodrug TH-302 inhibits tumor growth in preclinical models of cancer. Clin Cancer Res. 2012; 18:758-770. [PubMed: 22184053]

28. Supuran CT. Development of small molecule carbonic anhydrase IX inhibitors. BJU Int. 2008;101(Suppl 4): 39-40

29. Thiry A, Dogne JM, Masereel B, Supuran CT . Targeting tumor-associated carbonic anhydrase IX in cancer therapy. Trends Pharmacol Sci 2006; 27:566-573.

30. Trastour C, Benizri E, Ettore F, Ramaioli A, Chamorey E, Pouyssegur J, Berra E. HIF-1alpha and CA IX staining in invasive breast carcinomas: prognosis and treatment outcome. Int J Cancer 2007; 120:1451-1458.

31. V. M"uller, S. Riethdorf, B. Rack et al., "Prospective evaluation of serum tissue inhibitor of metalloproteinase 1 and carbonic anhydrase IXin correlation to circulating tumor cells in patients with metastatic breast cancer," Breast Cancer Research, vol. 13,no. 4, article R71, 2011.

32. Wigerup C, Påhlman S, Bexell D. Therapeutic targeting of hypoxia and hypoxia-inducible factors in cancer Pharmacology \& Therapeutics 2016; 152-169.

33. Wojtkowiak JW, Cornnell HC, Matsumoto S, et al. Pyruvate sensitizes pancreatic tumors to hypoxia-activated prodrug TH-302. Cancer Metabol. 2015; 3:2.

34. Wong, C.C.L., Zhang, H., Gilkes, D.M., et al., Inhibitors of hypoxia-inducible factor 1 block breast cancer metastatic niche formation and lung metastasis. J. Mol.Med., 2012; 90(7):803-815. [doi:10.1007/s00109-0110855-y]

35. Y. Lou, P. C. McDonald, A. Oloumi et al., "Targeting tumor hypoxia: suppression of breast tumor growth and metastasis by novel carbonic anhydrase IX inhibitors," Cancer Research, 2011;71: 3364-3376,

36. Zatovicova M, Jelenska L, Hulikova A, et al. Carbonic anhydrase IX as an anticancer therapy target: preclinical evaluation of internalizing monoclonal antibody directed to catalytic domain. Curr Pharm Des. 2010; 16:32553263. [PubMed: 20819068].

37. Zhang W, Shi X, Peng Y, Wu M, Zhang P, Xie R, et al. HIF-1alpha Promotes Epithelial-Mesenchymal Transition and Metastasis through Direct Regulation of ZEB1 in Colorectal Cancer. PLoS One. 2015; 10(6):e0129603. doi: 10.1371/journal.pone.0129603 PMID: 26057751

38. Zhang, H.,Wong, C. C.,Wei, H., Gilkes, D.M., Korangath, P., Chaturvedi, P et al., HIF-1-dependent expression of angiopoietin-like 4 and L1CAMmediates vascular metastasis of hypoxic breast cancer cells to the lungs. Oncogene 2012; 31:1757-1770. 